\title{
Monotonicity Properties of Oscillatory Solutions of Second Order Differential Equations
}

\author{
E. MÜLler-Pferfiger
}

Es wird untersucht, wie sich Monotonieeigenschaften der Koeffizienten gewöhńlicher Differentialgleichungen $\mathbf{z}$ weiter Ordnung auf oszillierende Lösungen $u$ sölcher Gleichungen übertragen. Zum Beispiel werdèn Aussagen gemacht über die Abstände der Nullstellen von $u, u^{\prime}$ und der von $u$ und $u^{\prime}$.untereinander.

Исследуется, как переносятся свонства монотониости коәффициентов обыкновенных пифференциальных уравненип второго порядка на осциллирующне решения $u$ такых уравнении. Делаются, например, высказывания, о расстоянии нулећ̆ функцй, $u, u^{\prime}$ и тех от $u$ и $u^{\prime}$ между собой.

It is proved in what way monotonicity properties of the coefficients of ordinary second order differential equations are transmitted to oscillatory solutions $u$ of such equations. For instance, there are statements on the distances of the zeros of $u, u^{\prime}$, and $u$ and $u^{\prime}$ mutually.

This paper generalizes the following theorem of P. Hartman and A. Wintiner [2]:' Consider the equation

$$
-u^{\prime \prime}+. Q(x)^{\prime} u=0, \quad x_{1} \leqq x \leqq x_{3}, \quad Q \in C, \quad Q \leqq 0,
$$

and let $u$ be a solution with three consecutive zeros $x_{1}, x_{2}, x_{3}$, and relative extrema at $\ddot{x}_{1}{ }^{\prime}, x_{1}<x_{1}{ }^{\prime}<x_{2}$, and $x_{2}{ }^{\prime}, x_{2}<x_{2}{ }^{\prime}<x_{3}$. Let $A$; be the area bounded by the $x$-axis, the straight line $x=x_{j}^{\prime}$, and the graph of $u$ belonging to the interval $\left[x_{j}, x_{j}{ }^{\prime}\right] ; j=1$, 2 , and let $A_{j}^{\prime}$ be the area bounded by the $x$-axis, the straight line $x=x_{j}^{\prime}$, and the graph of $u$ belonging to the interval $\left[x_{j}{ }^{\prime}, x_{j+1}\right], j=1,2$ (Fig. 1).

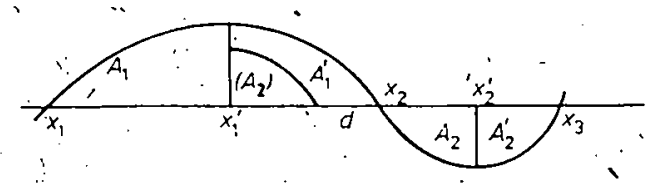

Fig. 1

If $Q$ is monotone decreasing on $\left[x_{1}, x_{3}\right]$; then $A_{j}^{\prime}$ can be placed into $A$, hy reflection at the straight line $x=x_{j}^{\prime}, j=1,2$, and $A_{2}$ can be placed into $A_{1}{ }^{\prime}$ by rotation through $180^{\circ}$ about the point $x_{2}$ and translation about $d=2 x_{2}-x_{1}{ }^{\prime}-x_{2}{ }^{\prime}$ to the left placing the abscissae of the extrema ordinates in coincidence. (After this rotation and translation of $A_{2}$ the new position of $A_{2}$ is denoted by $\left(A_{2}\right)$ in Fig. 1.) Concerning the monotonicity of the' quarter-waves $A_{j}, A_{j}^{\prime}$ and the half-waves $A_{j} \cup A_{j}^{\prime}$ if $Q$ is monotone decreasing or increasing compare also the papers of E. MakaI [9] and I. BIfrari [1].

In the following we consider the non-selfadjoint differential equations $(r, R \in$ $C[a, b])$

$$
-\left(P u^{\prime}\right)^{\prime}+R u^{\prime}+Q u=0 \quad\left(0<P \in C^{1}[a, b], 0>Q \in C[a, b]\right),
$$


and

$$
-\left(p v^{\prime}\right)^{\prime}+r v^{\prime}+q v=0 \quad\left(0<p \in C^{1}[a, b], 0>q \in C[a, b]\right) .
$$

Let $u$ be an oscillatory'solution to (1) and throughout this paper denote the zeros of $u$ by $x_{1}, x_{2}, \ldots$, and the zeros of $u^{\prime}$ being greater than $x_{1}$ by $x_{1}{ }^{\prime}, x_{2}{ }^{\prime}, \ldots$, so that $0 \leqq x_{1} \leq x_{1}{ }^{\prime}<x_{2}<x_{2}{ }^{\prime}<\ldots$. Define the areas $A_{j}, A_{j}{ }^{\prime}$ of the quarter-waves of $u$ as above. Further, let the following definitions hold throughout the paper.

i) $A_{j} \supseteq A_{j}^{\prime}$ means that $A_{j}^{\prime}$ can be placed into $A_{j}$ by reflection at the straight line $x=x_{i}^{\prime}$.

-ii) $A_{j-}^{\prime} \supseteqq A_{j+1}^{-}$means that $A_{j+1}$ can be placed into $A_{j}^{\prime}$ by rotation through $180^{\circ}$ about the point $x_{j+1}$ (Fig. 2 ; after the $180^{\circ}$-rotation the position of $A_{j+1}$ is denoted by , $\left.\left[A_{j+1}\right]\right)$.

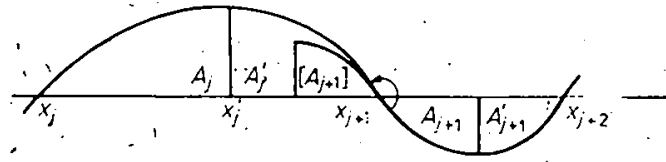

Fig. 2

iii) $A_{j} \supseteqq A_{j+1}$ means that $A_{j+1}$ can be placed into $A_{j}$ by reflection at the $x$-axis and translation about $x_{j+1}^{\prime}-x_{j}^{\prime}$, to the left.

iv) $A_{j}^{\prime} \supseteqq A_{j+1}^{\prime}$ means that $A_{j+1}^{\prime}$ can be placed into $A_{j}^{\prime}$ by reflection at the $x$-axis and translation about $x_{j+1}^{\prime}-x_{j}^{\prime}$ to the left.

' v) $A_{j} \cup A_{j}^{\prime} \supseteqq A_{j+1} \cup A_{j+1}^{\prime}$ means that $A_{j+1}^{\prime} \cup A_{j+1}^{\prime}$ can be placed into $A_{j} \cup A_{j}^{\prime}$ by reflection at the $x$-axis and translation about $x_{j+1}^{\prime}-x_{j}^{\prime}$ to the left.

'The inclusions $A_{j} \supset A_{j}^{\prime}, A_{j} \subseteq A_{j}^{\prime}, A_{j} \subset A_{j}^{\prime}$ and so on are analogously defined.

\section{Comparison theorems}

To compare oscillatory solutions of (1) and (2) these equations are to be transformed into Riccati differential equations.

L Lemma.1: Let u be a solution to (1) which doesn't vanish on $\left(t_{1}, t_{2}\right)$, and choose any positive function $\Phi \in C^{1}\left[t_{1}, t_{2}\right]$. Then the function

$$
y=-\Phi P u^{-1} u^{\prime}
$$

is a solution to the Riccati differential equation

$$
y^{\prime}=\Phi_{-1}^{-1} P^{-1} y^{2}+\left(\Phi^{-1} \Phi^{\prime}+P^{-1} R\right) y-\Phi Q \text { on } .\left(t_{1}, t_{2}\right)
$$

This assertion follows by an-easy calculation. Analogously, if $v$ /is a solution to (2) .which doesn't vanish on $\left(t_{1}, t_{2}\right)$ and $\varphi \in C^{1}\left[t_{1}, t_{2}\right]$ is any. positive function, then

$$
z=-\varphi p v^{-1} v^{\prime}
$$

is a solution to

$$
\therefore \quad \therefore \dot{z}^{\prime}=\varphi^{-1} p^{-1} z^{2}+\left(\varphi^{-1} \varphi^{\prime}+p^{-1} r\right) z-\varphi q \text { on }\left(t_{1} ; t_{2}\right)
$$

Theorem 1: Lët $u$ be a solution to (1) with $u(a)=u^{\prime}(c)=u(b)=0, a<c<b$, $u>0$ on $(a, b)$, and consider the solution $v$ to (2) determined by $v(c)=u(c), v^{\prime}(c)=0$. 
If there exist a number $\eta^{\prime}>0$ and a point $x_{0} \in[a, b]$ such that

$$
\begin{aligned}
& \eta P(x) \exp \left(-\int_{x_{0}}^{x} R P^{-1} d t\right) \geqq p(x) \exp \left(-\int_{x_{0}}^{x} r p^{-1} d t\right), \quad x \in\{a ; b], \\
& \eta Q(x) \exp \left(-\int_{x_{0}}^{x} R P^{-1} d t\right) \geqq q(x) \exp \left(-\int_{x_{0}}^{x} r p^{-1} d t\right), \quad x \in[a, b]
\end{aligned}
$$

then there exist points $\alpha \in[a, c)$ and $\beta \in(\dot{c}, b]$ with $v(\alpha)=0=v(\beta)$ and $0<\dot{v} \leqq$ on $(\alpha, \beta)$. If in (7) and (8) the signs $\geqq$ are replaced by $\leqq$ then $v \geqq u$-on $[a, b]$. If in (7) and (8) at least one of the signs $\geqq$ is replaced by $>$, then $\alpha \in(a, c), \beta \in(c, b)$ and $0<v$ $<u$ on $(\alpha, c) \cup(c, \beta)$. If in (7) and (8) the signs $\leqq$ are valid and at least one of the arising inequalities, is strict, then $v>u$ on $[a, c) \cup(c, b]$.

Proof: By setting

and

$$
\Phi(x)=\eta \exp \left(-\int_{x_{0}}^{x} R P^{-1} d t\right), \quad x \in[a, b],
$$

$$
\varphi(x)=\exp \left(-\int_{x_{0}}^{x} r p^{-1} d t\right), x \in[a, b]
$$

it follows from (4) and (6) that

$$
y^{\prime}=\Phi^{-1} P^{-1} y^{2}-\Phi Q \quad \text { on } \quad(a, b) ; y(c) \doteq 0,
$$

and

$$
z^{\prime}=\varphi^{-1} p^{-1} z^{2}-\varphi q, \quad z(c)=0,
$$

respectively. (12) holds on every interval $\left(t_{1}, t_{2}\right), c \in\left(t_{1} ; t_{2}\right)$, where $z$ exists. By (7) and (8) we obtain $z^{\prime} \geqq y^{\prime}$ everywhere in the strip $S_{\left(t_{1}, t_{2}\right)}=\left\{(x, y) \mid x \in\left(t_{1}, t_{2}\right)\right.$, $y \in(-\infty, \infty)\}$. Thus, concerning the solution to (11) and (12) it follows that $z \leqq y$ on $\left(t_{1}, c\right]$ and $z \geqq y$. on [c, $\left.t_{2}\right)$ (cf. [3, p: 91]). Therefore, and by $y(x) \rightarrow-\infty$ as $x \downarrow a$, $y(x) \rightarrow+\infty$ as $x \uparrow b$ it follows that there exist points, $\alpha \cdot \in[a, c)$ and $\beta \in(c, b]$ such that $z(x) \rightarrow-\infty$ as $x \downarrow \alpha, z(x) \rightarrow+\infty$ as $x \uparrow \beta$. Hence, we have $v(\alpha)=0=v(\beta)$. Further, $y^{\prime}>0$ holds on $(\alpha, \beta)$ because of (11) and $Q<0$. This implies $z \leqq y<0$ on $(\alpha, c)$ and $0<y \leqq z$ on $(c, \beta)$. Thus, by (3), (5), (7), and (8), we obtain $v^{-1} v^{\prime} \geqq u^{-1} u^{\prime}$ $>0$ on $(\alpha, c)$ and $v^{-1} v^{\prime} \leqq u^{-1} u^{\prime}<0$ on $(c, \beta)$. Finally, integration leads to $v \leqq u$ on $[\alpha, \beta]$. If in (7) and (8) the signs $\geqq$ are replaced by the signs $\leqq$ the assertion $v \geqq u$ on $[a, b]$ can analogously be proved.

Assume now that in (7) or (8) at least one of the signs $\geqq$ is replaced by $>$. Then, in 'view of (11) and (12), it follows that $z^{\prime}>y^{\prime}$ everywhere in the strip $S_{\left(t_{1}, t_{1}\right)}$ whenever $(x, y) \neq(c, y)$. Thus; we obtain $z<y<0$ on $(\alpha, c), 0<y<z$ on $(c, \beta)$, and, consebuently, $0<v<u$ on $(\alpha, c) \cup(c, \beta)$. To prove that $\beta \in(c, b)$, for instance, choose a point $x_{1} \in(c, \beta)$ and consider the solution $y_{1}$ to $(11)$ determined by $y_{1}\left(x_{1}\right)=z\left(x_{1}\right)$ $\left(>y\left(x_{1}\right)\right)$. The function $w=\left(y_{1},-y\right)^{-1}$ is a solution to

$$
w^{\prime}+2 y \Phi^{-1} P^{-1} w+\Phi^{-1} P^{-1}=0 \text {, on }(a, b)
$$


(cf. $[3,10])$. Hence

$$
\begin{aligned}
w(x) & =\exp \left(-2 \int_{x_{1}}^{x} y \Phi^{-1} P^{-1} d t\right)\left(w\left(x_{1}\right)-\int_{x_{1}}^{x} \Phi^{-1} P^{-1} \exp \left(2 \int_{x_{1}}^{t} y \Phi^{-1} P^{-1} d \tau\right) d t\right) \\
& =\frac{u^{2}(x)}{u^{2}\left(x_{1}\right)}\left(w\left(x_{1}\right)-u^{2}\left(x_{1}\right) \int_{x_{1}}^{x} \Phi^{-1} P^{-1} u_{1}^{-2} d t\right) \\
\vdots & \vdots \\
w\left(x_{1}\right) & =\left(\dot{y}_{1}\left(x_{1}\right)-y\left(x_{1}\right)\right)^{-1}>0 .
\end{aligned}
$$

It follows from. (14)'and

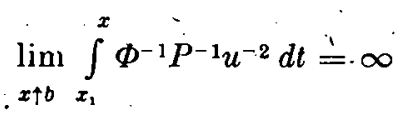

that there exists a point $\xi_{1} \in\left(x_{1}, b\right)$ such that $w(x) \rightarrow 0$ as $x \uparrow \xi_{1}$. Hence, we have $y_{1}(x) \rightarrow \infty$ as $x \uparrow \xi_{1}$. Since $z^{\prime} \geq y_{1}^{\prime}$ in $S_{\left(t_{1}, t_{2}\right)}$; it follows that $z>y_{1}$ on $\left(x_{1}, t_{2}\right)$. Therefore, there exists a point $\beta \in(c, b)$ such that $z(x) \rightarrow \infty$ as $x \uparrow \beta$. This proves that $v(\beta)=0, \beta \in(c, b)$. The assertion $\alpha \cdot e(a, c)$ can analogously be proved

In the selfadjoint case $R \equiv r \equiv \mathbf{0}$ concerning the location of the zero $\beta$ of $v$ Theorem 1 is essentially a result by LEIGHTON [5].

Theorem 2: Let $u$ be a solution to (1) with $u(a)=u^{\prime}(b)=0, u^{\prime}>0$ on $[a, b)$ and consider the solution $v$ to $(2)$ determined by $v(a)=0, v^{\prime}(a)=u^{\prime}(a)>0$. If there exist $a$ number $\eta>0$ and $a$ point $x_{0} \in[a, b]$ such that (7) and (8) are fulfilled, then there exists a point $\beta \in(a, b]$ with $v^{\prime}(\beta)=0$ and $v^{\prime \prime}>0$ on $[a, \beta)$. If in (7) and (8) the signs $\geqq$ are replaced by $\leqq$, then $v^{\prime}>0$ on $[a, b)$. If (7) and (8) hold and one of these inequalities' is strict, then $\beta \in(a, b)$.

- Proof: Use the functions (9), (10), and, consequently, the equations (11) and (12). It follows from the hypotheses of the theorem that $y(x) \rightarrow-\infty$ and $z(x) \rightarrow-\infty$ as $x \downarrow a ; y^{\prime}>0$ on $(a, b), y(b)=0$, and $y<0$ on $(a, b)$. Iet the conditions (7) and (8) be fulfilled. We prove that $z \geqq y$ on all intervals $\left(a, \beta^{\prime}\right), \dot{\beta}^{\prime} \leqq b$, where $z$ exists. Assuming the contrary suppose that there exists a point $x_{1} \in\left(a, \beta^{\prime}\right)$ with $z\left(x_{1}\right)<y\left(x_{1}\right)$. Consider the solution $y_{1}$ to (11) determined by $y_{1}\left(x_{1}\right)=z\left(x_{1}\right)$. The function $w=\left(y_{1}-y\right)^{-1}$ is a solution to (13). $w$ is given by (14) with $w\left(x_{1}\right)=\left(y_{1}\left(x_{1}\right)-y\left(x_{1}\right)\right)^{-1}<0$ and we' have

$$
\lim _{x \downarrow a} \int_{x_{1}}^{x} \Phi^{-1} P^{-1} \mu^{-2} d t=-\infty .
$$

Hence, there exists a point $\xi_{1} \in\left(a, x_{1}\right)$ such that $w(x) \rightarrow 0$ as $x \downarrow \xi_{1}$ and, consequently, $y_{1}(x) \rightarrow-\infty$ as $x \downarrow \xi_{1}$. Because of $y^{\prime} \leqq z^{\prime}$ everywhere in the strip $S_{\left(a, \beta^{\prime}\right)}=\{(x, y)$ $\left.\mid x \in\left(a, \beta^{\prime}\right), y \in(-\infty, \infty)\right\}$ we obtain $z \leqq y_{1}$ on the left-hand side of $x_{1}$. Hence, there exists a point $\xi_{2} \in\left[\xi_{1}, x_{1}\right)$ with $z(x) \rightarrow-\infty$ as $x \downarrow \xi_{2}$. This, however, contradicts the fact that $z$ exists on $\left(a, \beta^{\prime}\right)$. This proves $z \geqq y$ on $\left(a, \beta^{\prime}\right)$. Since $y(b)=0$, there exists a point $\beta \in(a, b]$ with $z(\beta)=0$ and $-\infty<y \leqq z<0$ on $(a, \beta)$. Thus, we obtain $v^{\prime}(\beta)=0$ and $v^{\prime}>0$ on $[a, \beta)$. If $(7)$ and $(8)$ hold, but one of these inequalities is strict, we obtain $y^{\prime}<z^{\prime}$ in $S_{(a, \beta)}$ and, consequently, $y<z$ on $(a, \beta)$. It now follows from $y^{\prime}<z^{\prime}$ on $(a, \beta)$ that $\beta<b$.

If (7) and (8) with $\leqq$ in place of $\geqq$ are considered, we prove that $z \leqq y$ on $(a, b]$. Assume the contrary and let $x_{1} \in(a, b)$ be a point with $z\left(x_{1}\right)>y\left(x_{1}\right)$. The solution $y_{1}$ to (11) determined by $y_{1}\left(x_{1}\right)=z\left(x_{1}\right)$ is monotone increasing on $\left(a, x_{1}\right]$. The case $y_{1}(x)$ $\rightarrow-\infty$ as $x \downarrow a$ is impossible, because, by assuming this case, it would follow that there exists a point $\xi_{1} \in\left(a, x_{1}\right)$ with $y(x) \rightarrow-\infty$ as $x \downarrow \xi_{1}$ as it is seen from above. 
Hence, we have $y_{1}(x) \rightarrow \omega$ as $x \downarrow a, \omega \in\left(-\infty, y_{1}\left(x_{1}\right)\right)$. Further, by the hypotheses under consideration, it follows that $y^{\prime} \geqq z^{\prime}$ in the strip $S_{(a, b)}$ which implies that $z \geqq y_{1}>\omega$ on $\left(a, x_{1}\right]$. This, however, is impossible because of $z(x) \rightarrow-\infty$ as $x \downarrow a$. Hence, we have $z \leqq y$ on $(a, b]$. This proves that $v_{1}^{\prime}>0$ on $[a, b)$

In the selfadjoint case $R \equiv r \equiv 0$ and $\eta=1$ Theorem 2 is due to Leígiton [5, 6]. The next theorem can similarly be proved.

Theorem 3: Let $u$ be a solution to (1) with $u(b)=u^{\prime}(a)=0, u^{\prime}<0$ on $(a, b]$, and consider the solution $v$ to $(2)$ determined by $v(b)=0, v^{\prime}(b)=u^{\prime}(b)<0$. If there exist $a$ number $\eta>0$ and a point $x_{0} \in[a, b]$ such that (7) and (8) are satisfied, then there exists a point $\alpha \in[a, b)$ with $v^{\prime}(\alpha)=0$ and $v^{\prime}<0$ on $(\alpha, b]$. If, additionally, one of these inequalities is strict, then $\alpha \in(a, b)$. If in (7) and (8) the signs $\geqq$ are replaced by $\leqq$, then $v^{\prime}<0$ on $(a, b)$.

By setting $P \cong p \equiv \Phi \equiv \varphi \equiv 1$ in (4) and (6) we obtain the Riccati equations.

$$
y^{\prime}=y^{2}+R y-Q, \quad y=-u^{-1} u^{\prime}
$$

and

$$
z^{\prime}=z^{2}+r z-q, \quad z=-v^{-1} v^{\prime},
$$

which lead to the following theorem.

- Theorem 4: Let $u$ and $v$ be solutions to

$$
-u^{\prime \prime}+R u^{\prime}+Q u=0, \quad(R, Q \in C[a, b], Q<0)
$$

and

$$
-v^{\prime \prime}+r v^{\prime}+q v \stackrel{\rho}{=} 0, \quad(r, q \in C[a, b], q<0)
$$

with $u(a)=v(a)=u^{\prime}(b)=0, u^{\prime}(a)=v^{\prime}(a)>0, u^{\prime}>0$ on $[a, b)$. If

$$
r \leqq R, \quad q \leqq Q \quad \text { on }[a, b],
$$

then there exists a point $\dot{\beta} \in(a, b]$ with $v^{\prime}(\beta)=0, v^{\prime}>\dot{0}$ on $[a, \beta)$, and $0 \leqslant v \leqq \dot{u}$ on $(a, \beta]$. The point $\beta$ is equal to $b$ only if the equations (17) and (18) are identical. If.

$$
r \geqq R, \quad q \geqq Q \quad \text { on }[a, b],
$$

then $v \geqq u$ on $[a, b], v^{\prime}>0$ on $[a, b)^{\prime}$, and $v^{\prime}(b)=0$ only if (17) and (18) are identical.

Proof:- The function $y=-u^{-1} u^{\prime}$ is defined on $(a, b j$. By the hypotheses on $u$ it follows that $y(x) \rightarrow-\infty$ as $x \downarrow a ; y(b)=0$, and $y<0$ on $(a, b)$. Let the hypothesis (19) be fulfilled. Assume that there doesn't exist a zero of $v^{\prime}$ on $(a, b)$. Then the function $z=-v^{-1} v^{\prime}$ is negative on $(a, b)$. By (15), (16), and (19) it follows that $y^{\prime} \leqq z^{\prime}$ everywhere in the half-strip $H_{(a, b)}=\{(x, y) \mid x \in(a, b), y \in(-\infty, 0)\}$ : Thus, as in the proof of Theorem 2, we obtain $y \leqq z$ on intervals $\left(a, \beta^{\prime}\right], \beta^{\prime} \in(a, b]$, where $-\infty$ $<z \leqq 0$. If the functions $y$ and $z$ are not identical, there exists a point $x_{1} \in(a, b)$ with $y\left(x_{1}\right)<z\left(x_{1}\right)(<0)$. The solution $y_{1}$ to (15) determined by the initial value $y_{1}\left(x_{1}\right)=z\left(x_{1}\right)$ must cross the $x$-axis because of $y(b)=0$ and the uniqueness of solutions to $(15) \cdot$ Now, it follows from $y^{\prime} \leqq z^{\prime}$ in $H_{(a, b)}$ that $y_{1} \leqq z$ to the right of $x_{1}$ and for points $\left(x, y_{1}\right)$ and $(x, z)$ which are placed in $H_{(a, b)}$. Hence, the graph of $z$ must also cross the $x$-axis. This, however, contradicts the assumption that $v^{\prime}$ does not vanish on $(a, b)$. Hence, we have $y=z$ on $(a, b)$. Thus, by (15), (16), and (19) it follows that $Q \equiv q$ and $R \equiv r$, i.e. the equations (17) and (18) are identical if $v^{\prime}$ does not vanish on $(a, b)$. Let $\beta \in(a, b]$ be the first zero of $v^{\prime}$. Then we have $y \leqq z<0$ on $(a, \beta)$, which implies that $u^{-1} u^{\prime} \geqq v^{-1} v^{\prime}>0$ on $(\dot{a}, \beta)$. By integration we obtain $0<v \leqq u$ on $(a, \beta]$. 
This proves the first part of the theorem. If $(20)$ is supposed exchange the parts of the equations (17) and (18)

The different parts of the following theorem can analogously be proved.

Theorem 5: Consider the differential equations (17) and (18) on $[a, b]$.

i) Let $u$ and $v$ be solutions to. (17) and (18), respectively, with $u(a)=v(a)>0$, ${ }^{\prime} u^{\prime}(a)=v^{\prime}(a)=u(b)=0, u>0$ on $[a, b)$. If

$$
r \geqq R, \quad q \leqq Q<0 \text { on }[a, b] \text {, }
$$

then $u^{\prime}<0$ on $[a, b]$, and there exists a point $\beta \in(a ; b]$ such that $v(\beta) \doteq 0,0<v \leqq u$ on $[a ; \beta) ;$ and $v^{\prime}<0$ on (a, $\left.\beta\right]$, where $\beta$ is equal to $b$ only if (17) and (18) are identical. $I$,

$$
r \leqq R, \quad Q \leqq q<0 \text { on }[a, b]
$$

then $v \geqq u$ on $[a, b]$, where $v(b)=0$ only if (17) and (18) are identical:

ii) Let $u$ and $v$ be solutions to (17), and (18), respectively, with $u(b)=v(b)>0$, $u^{\prime}(b)=v^{\prime}(b)=u(a)=0$, and $u>0$ on $(a, b]$. If

$$
r \leqq R, \quad q \leqq Q<0 \quad \text { on }[a, b],
$$

then $u^{\prime}>0$ on $[a, b]$ and there exists a point $\alpha \in[a, b)^{\prime}$ such that $v(\alpha)=0,0<v \leqq u$ on $(\alpha, b]$ and $v^{\prime}>0$ on $[\alpha, b)$, where $\alpha$ is equal to a only if (17) and (18) are identical. If

$$
r \geqq R, \quad Q \leqq q<0 \quad \text { on }[a, b]
$$

then $v \geqq u$ on $[a, b]$, where $v(a) \doteq \dot{0}$ only if (17) and (18) are identical.

iii) Let $u$ and $v$ be solutions to (17). and (18), respectively, with $u(b)=v(b)=u^{\prime}(a)$ $=0, u^{\prime}(b)=v^{\prime}(b)<0, u^{\prime}<0$ on $(a, b]$. If

$$
r \geqq R, \quad q \leqq Q<0 \text { on }[a, b],
$$

then there exists a point $\alpha \in[a, b)$ with $v^{\prime}(\alpha)=0,0<v \leqq u$ on $[\alpha, b)$ and $v^{\prime}<0$ on $(\alpha, b]$, where $\alpha$ is equal to $a$ only if (17) and (18) are identical. If

$$
r \leqq R, \quad Q \leqq q<0 \text { on }[a, b]
$$

then $v>u$ on $[a, b], v^{\prime}<0$ on $(a, b]$, and $v^{\prime}(a) \doteq 0$ only if (17) and (18) are identical.

Proof: i) The assertion $u^{\prime}<0$ on $(a, b]$, for instance, easily follows from (15): Since $y(a)=0, y^{\prime}(a)=-Q(a)>0$, the function $y$ is positive in a neighbourhood of $a$. Further, the graph of $y$ cannot touch the $x$-axis at a point $x_{0} \in(a, b)$ as can be proved as follows. Assume that $x_{0}$ is the smallest point to the right of $a$ with $y\left(x_{0}\right)=0$. Since $y>0$ on $\left(a, x_{0}\right)$, we have $y^{\prime}\left(x_{0}\right) \leqq 0$, contradictory to $y^{\prime}\left(x_{0}\right)=-Q\left(x_{0}\right)>0$. $y>0$ on $(a, b)$ implies $u^{\prime}<0$ on $(a, b)$. To prove the other assertions of the theorem compare the proof of Theorem 4

Of course, the Theorems 4 and 5 can easily be applied to the selfadjoint equations (1) and (2) with $R \equiv 0$ and $r \equiv 0$, respectively, to obtain analogous comparison theorems.

\section{Monotonicity properties of solutions}

In the following the comparison theorems from above are used to study monotonicity properties of oscillatory solutions of second order differential equations implied by corresponding monotonicity behaviour of the coefficients of the differential equations. 
Theorem 6: Let $u$ be an oscillatory solution to (1) on $[0, \infty)$. Denote the zeros of $u$ । by $x_{1}, x_{2}, \ldots$, and the zeros of $u^{\prime}$ by $x_{1}^{\prime}, x_{2}{ }^{\prime}, \ldots$, so that $0 \leqq x_{1}<x_{1}{ }^{\prime}<x_{2}<x_{2}{ }^{\prime}<\ldots{ }^{\prime}$ ) If there exists $c \in \mathbb{R}$ such that the functions

$$
P(x) \exp \left(c x-\int_{0}^{x} R P^{-1} d t\right), Q(x) \exp \left(c x-\int_{0}^{x} R P^{-1} d t\right)
$$

are monotone decreasing (increasing) on $[0, \infty)$, then, for $j \in \mathbb{N}$,

$$
x_{j}^{\prime}-x_{j} \text { (引) } \dot{x}_{j+1}^{\prime}-x_{i+1}, x_{j+1}-x_{j}^{\prime}\left(\geqq x_{j+2}-x_{j+1}^{\prime}\right.
$$

and, consequently,,

$$
\dot{x}_{j+1}-x_{j}(\underline{\underline{z}}) x_{j+2}-x_{j+1}, \quad x_{j+1}^{\prime}-x_{j}^{\prime}(\underline{\underline{z}}) x_{j+2}^{\prime}-x_{j+1}^{\prime} .
$$

If additionally, one of the functions (22) is strictly monotone, the inequalities (23) and(24) are also strict.

Proof: The function $\tilde{u}(x)=u\left(x_{j+1}^{\prime}-x_{j}^{\prime}+x\right)\left(x \in\left[x_{j}, x_{j+1}\right] ; j \in \dot{\mathbf{N}}\right)$ is a solution to the differential equation

$$
-\left(P\left(x_{j+1}^{\prime}-x_{j}^{\prime}+x\right) \bar{u}^{\prime}\right)^{\prime}+\dot{R}\left(x_{j+1}^{\prime}-x_{j}^{\prime}+x\right) \bar{u}^{\prime}+Q\left(x_{j+1}^{\prime}-x_{j}^{\prime}+x\right) \bar{u}=0 .
$$

Assume that the functions (22) are monotone decreasing. Then

$$
\begin{aligned}
& \eta_{i} P(x) \exp \left(c x-\int_{0}^{x} R P^{-1} d t\right) \\
& \geqq \eta_{j} P\left(x_{j+1}^{\prime}-x_{j}^{\prime}+x\right) \exp \left(c\left(x_{j+1}^{\prime}-x_{j}^{\prime}+x\right)-\int_{0}^{x_{j+1}^{\prime}-x_{j}^{\prime}+x} R P^{-1} d t\right) \\
& =P\left(x_{j ! 1}^{\prime}-x_{j}^{\prime}+x\right) \exp _{s}\left(c x-\int_{0}^{x} R\left(x_{j+1}^{\prime}-x_{j}^{\prime}+\tau\right) P^{-1}\left(x_{j+1}^{\prime}-x_{j}^{\prime}+\tau\right) d \tau\right)
\end{aligned}
$$

with $\eta_{j}=\exp \left(c\left(x_{j}^{\prime}-x_{j+1}^{\prime}\right)^{-}+\int_{0}^{x_{j+1}^{\prime}-x_{j}^{\prime}} \cdot R P^{-1} d t\right)$. Hence,

$$
\begin{aligned}
& \tilde{\eta}_{j} P(x) \exp \left(-\int_{x_{j}^{\prime}}^{x} R P^{-1} d t\right) \\
& \geqq P\left(x_{j+1}^{\prime}-x_{j}^{\prime}+x\right) \exp \left(-\int_{x_{j}}^{x} R\left(x_{j+1}^{\prime}-x_{j}^{\prime}+\tau\right) P^{-1}\left(x_{j+1}^{\prime}-x_{j}^{\prime}+\tau\right) d \tau\right),
\end{aligned}
$$

'where

$$
\tilde{\eta}_{j}=\ddot{\eta}_{j} \exp \left(-\int_{0}^{x_{j}^{\prime}} R P^{-1} d t+\int_{0}^{x_{j}^{\prime}} R\left(x_{j+1}^{\prime}-x_{j}^{\prime}+\tau\right) P^{-1}\left(x_{j+1}^{\prime}-x_{j}^{\prime}+\tau\right) d \tau\right)
$$

1) Here and in the following a possible zero $x_{0}^{\prime} \in\left[0, x_{1}\right)^{\prime}$ of $u^{\prime}$ is disregarded. 
$x \in\left[x_{j}, x_{j+1}\right], j \in \mathbb{N}$. Analogously,

$$
\begin{aligned}
& \eta_{j} Q(x) \exp \left(\div \int_{x_{j}}^{x} R P^{-1} d t\right), \\
& \geqq Q\left(x_{j+1}^{\prime}-x_{j}^{\prime}+x\right) \exp \left(-\int_{x_{1}}^{x} R\left(x_{j+1}^{\prime}-x_{j}^{\prime}+\tau\right) P^{-1}\left(x_{j+1}^{\prime}-x_{j}^{\prime}+\tau\right) d \tau\right),
\end{aligned}
$$

$x \in\left[x_{j}, x_{j+1}\right], j \in \mathbb{N}$. To finish the first part of Theorem 6 set

$$
\begin{aligned}
& P\left(x_{j+1}^{\prime}-x_{j}^{\prime}+x\right)=p(x), \quad R\left(x_{j+1}^{\prime}-x_{j}^{\prime}+x\right)=r(x), \\
& Q\left(x_{j+1}^{\prime}-x_{j}^{\prime}+x\right)=q(x), \quad v(x)=u\left(\dot{x}_{j}^{\prime}\right) \dot{u}^{-1}\left(x_{j}^{\prime}\right) \tilde{u}(x), \\
& \bar{x}_{j}=c=x_{0}, \quad x_{j}=a, \quad x_{j+1}=b,
\end{aligned}
$$

and apply Theorem 1 . The part of Theorem 6 described by the brackets can analogously-be proved. If, additionally, one of the functions (22) is strictly monotone decreasing (increasing), the remaining part of Theorem 6 follows from the last part of Theorem 1

In the special case $P \equiv 1 ; R \equiv 0, c=0$ and concerning the inequalities $x_{j+1}^{\prime}-x_{j}^{\prime}$ (<) $x_{j+2}^{\prime}-x_{j+1}^{\prime}$. Theorem 6 is due to A. Laforgia [4].

Theorem $7:$ Let $u$ be an oscillatory solution to $(1)$ on $[0 ; \infty)$. If the functions

$$
P(x) \exp \left(-\int_{0}^{x} R P^{-1} d t\right), Q(x) \exp \left(-\int_{0}^{x} R P^{-1} d t\right)
$$

are monotone decreasing (incréasing) on $[0, \infty),{ }^{\prime}$ then $x_{j}^{\prime}-x_{j}\left(\sum_{\underline{\underline{y}}} x_{j+1}-x_{j}^{\prime}\left(\sum_{1} x_{j+1}^{\prime}\right.\right.$ 1. $\quad-\quad-x_{j+1}$ and $A_{j}$ 产, $A_{j}^{\prime}(j \in \mathbb{N})$. $^{\prime} I f$, additionally, one of the functions $(25)$ is strictly monotone, decreasing (increasing), then the asserted inequalities and inclusions are also strict.

Proof: We prove that $A_{j} \supseteqq A_{j}{ }^{\prime}$ if the functions (25) are monotone decreasing. The function $\bar{u}(x)=u\left(2 x_{j}{ }^{\prime}-x\right), x \in\left[x_{j} ; x_{j}{ }^{\prime}\right]$, is a solution to the differential equation

$$
-\left(P\left(2 x_{j}^{\prime}-x\right) \tilde{u}^{\prime}\right)^{\prime}-R\left(2 x_{j}^{\prime}-x\right) \tilde{u}^{\prime}+Q\left(2 x_{j}^{\prime}-x\right) \bar{u}=0
$$
with $\vec{u}\left(x_{j}{ }^{\prime}\right)=u\left(x_{j}{ }^{\prime}\right), \tilde{u}^{\prime}\left(x_{j}{ }^{\prime}\right)^{\prime}=-u^{\prime}\left(x_{j}{ }^{\prime}\right)=0$. Since $P(x) \exp \left(-\int_{0}^{x} R P^{-1} d t\right)$ is mono-
tone decreasing, we have

$$
\begin{aligned}
& P(x) \exp \left(-\int_{x^{\prime}}^{x} R P^{-1} d t\right)=\exp \left(\int_{0}^{x_{0}^{\prime}} R P^{-1} d t\right) P(x) \exp \left(-\int_{0}^{x} R P^{-1} d t\right) \\
& \geqq \exp \left(\int_{0}^{x^{\prime}} R P^{-1} d t\right) P\left(2 x_{j}^{\prime}-x\right) \exp \left(-\int_{0}^{2 x_{j}^{\prime}-x} R P^{-1} d t\right) \\
& =P\left(2 x_{i}^{\prime}-x\right) \exp \left(-\int_{x_{j}^{\prime}}^{2 x_{j}^{\prime}-x} R P^{-1} d t\right) \\
& =P\left(2 x_{j}^{\prime}-x\right) \exp \left(-\int_{x_{i}^{\prime}}^{x}\left(-R\left(2 x_{j}^{\prime}-\tau\right)\right) P^{-1}\left(2 x_{j}^{\prime}-\tau\right) d \tau\right) \text {, }
\end{aligned}
$$


$x \in\left[x_{j}, x_{j}^{\prime}\right]$. Analogously

$Q(x) \exp \left(-\int_{x^{\prime}}^{x} R P^{-1} d t\right) \geqq Q\left(2 x_{i}^{\prime}-x\right) \exp \left(-\int_{x_{j}^{\prime}}^{x}\left(-R\left(2 x_{j}^{\prime}-\tau\right)\right) P^{-1}\left(2 x_{j}^{\prime}-\tau\right) d \tau\right)$,

$x \in\left[x_{j}, x_{j}^{\prime}\right]$. By setting

$$
\begin{aligned}
& P\left(2 x_{j}^{\prime}-x\right)=p(x), \quad-R\left(2 x_{j}^{\prime}-x\right)=r(x), \quad Q\left(2 x_{j}^{\prime} \dddot{\prime}^{\prime} x\right)=q(x), \\
& \tilde{u}(x)=v(x), \quad x_{j}^{\prime}=x_{0}=c, \quad x_{j}=a, \eta=1
\end{aligned}
$$

and applying Theorem 1 we obtain $A_{j}^{\prime} \leqq A_{j}$ and, consequently, $x_{j}^{\prime}-x_{j} \geqq x_{j+1}-x_{j}^{\prime}$. To prove $x_{j+1}-x_{j}^{\prime} \geqq x_{j+1}^{\prime}-x_{j+1}$ define the function $\bar{u}(x)=-u\left(2 x_{j+1}-x\right), x \in\left[x_{j}^{\prime}\right.$, $\left.\cdot x_{j+1}\right]$. This function is a solution to

$$
-\left(P\left(2 x_{j+1}-x\right) \bar{u}^{\prime}\right)^{\prime}-R\left(2 x_{j+1}-x\right) \bar{u}^{\prime}+\dot{Q}\left(2 x_{j+1}-x\right) \bar{u}=0
$$

with $\tilde{u}\left(x_{j+1}^{-}\right)=-u\left(x_{i+1}\right)=0, \tilde{u}^{\prime}\left(x_{j+1}\right)=u^{\prime}\left(x_{j+1}\right)$. Now; conclude as above and apply Theorem 3 . Thus, we obtain $x_{j+1}-\dot{x}_{j}^{\prime} \geqq x_{j+1}^{\prime}-x_{j+1}$. If, additionally, one of the functions (25) is strictly monotone decreasing, by using the Theorems 1 and 3 the corresponding assertions can analogously be proved. The same holds in the case that the functions (25) are monotone increasing

- Theorem 8: Consider the differential equation

$$
-u^{\prime \prime}+R u^{\prime}+Q \dot{Q}=0 \quad(R, Q \in C[0, \infty), Q<0)
$$

and let $u$ bé an 'oscillatory solution.

i) If the coefficients are monotone decreasing ${ }^{4}\left(\right.$ increasing) on $[0, \infty)$, then $x_{j}^{\prime}-x_{j}$ (3) $x_{j+1}^{\prime}-x_{j+1} ; j \in \mathbb{N}: I f$, additionally, one of these coefficients is strictly decreasing (increasing), then $x_{j}^{\prime}-x_{i}(<) x_{j+1}^{\prime}-x_{j+1}, j \in \mathbb{N}$.

ii) If $R$ is monotone decreasing (increasing) and $Q$ is monotone increasing (decreasing), then $x_{j+1}-x_{j}^{\prime}\left(\sum_{1} x_{j+2}-x_{j+1}^{\prime}, j \in \mathbb{N}\right.$. If, additionally, one of these functions is strictly monotone, then $x_{j+1}-x_{j}^{\prime}\left(x_{j+2}-x_{j+1}^{\prime}, j \in \mathbb{N}\right.$.

Proof: Let $R$ and $Q$ be monotone decreasing on $[0, \infty)$. The solution $v \doteq u^{-1}\left(x_{j+1}^{\prime}\right)$ $\times u\left(x_{j}{ }^{\prime}\right) u$ to $(26)$ has the properties $v\left(x_{j+1}^{\prime}\right)=u\left(x_{j}^{\prime}\right)$ and $v^{\prime}\left(\dot{x}_{j+1}^{\prime}\right)=u^{\prime}\left(x_{j}{ }^{\prime}\right)=0$. By translating the graphs of $v, R$, and $Q$ belonging to the interval $\left[x_{j+1}, x_{j+1}^{\prime}\right]$ to the left about $x_{j+1}^{\prime}-x_{j}^{\prime}$ and applying Theorem $5 /$ ii), we obtain $x_{j}^{\prime}-x_{j} \geqq x_{j+1}^{\prime}-x_{j+1}$. Additionally, we have $x_{j}^{\prime}-x_{j}>x_{j+1}^{\prime}-x_{j+1}^{\prime}$ if one of the coefficients, is strictly decreasing. By the help of Theorem 5 the other assertions of Theorem 8 can analogously be proved

Theorem 9: Let $u$ be an oscillatory solution to (26) and as in the introduction denote the areas of the quarter-waves by $A_{j}$ and $A_{j}{ }^{\prime}$, respectively.

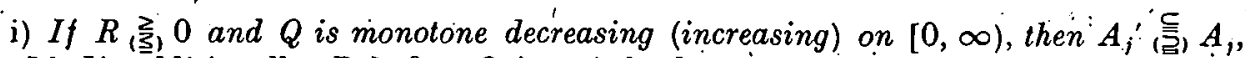
$j \in \mathbb{N}$. If, additionally; $R \underset{k}{\gtrless} 0$ or $Q$ is strictly decreasing (increasing), then $A_{j}^{\prime}\left(\vec{c}, \dot{A}_{j}\right.$, $j \in \mathbb{N}$.

ii) If $R$ (ș 0 and $Q$ is monotone decreasing (increasing) on $[0, \infty)$, then $A_{j}{ }^{\prime}$ (忌) $A_{i+i}$;

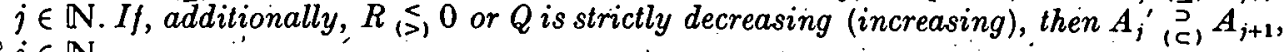
$j \in \mathbb{N}$. 
Proof: Assume first that $R \geqq 0$ and $Q$ is monotone decreasing on $[0, \infty)$. The func: tion $\bar{u}(x)=u\left(2 x_{j}{ }^{\prime}-x\right), x \in\left[x_{i}, x_{j}{ }^{\prime}\right]$, is a solution to the differential equation $-\tilde{u}^{\prime \prime}$ $-R\left(2 x_{j}^{\prime}-x\right) \bar{u}^{\prime}+Q\left(2 x_{j}^{\prime}-x\right) \bar{u}=0$ with the initial values $\tilde{u}\left(x_{j}^{\prime}\right)=u\left(x_{j}{ }^{\prime}\right), \bar{u}^{\prime}\left(x_{j}^{\prime}\right)$ $=-u^{\prime}\left(x_{j}{ }^{\prime}\right)=0$. Hence, by Theorem $\left.5 / \mathrm{ii}\right)$, we have $\bar{u} \leqq u$ on $\left[2 x_{j}{ }^{\prime}-x_{j+1}, x_{j}{ }^{\prime}\right]$ if $-R\left(2 x_{j}^{\prime}-x\right) \leqq R(x)$ and $Q\left(2 x_{j}{ }^{\prime}-x\right) \leqq Q(x), x \in\left[x_{j}, x_{j}^{\prime}\right]$. But, in the present case, these conditions are satisfied.

We else discuss the case $R \leqq 0$ and $Q$ is monotone decreasing. The function $u(x)$ $=-u\left(2 x_{j+1}-x\right), x \in\left[x_{j}{ }^{\prime}, x_{j+1}\right]$, is a 'solution to the differential equation

$$
-\bar{u}^{\prime \prime}-R\left(2 x_{j+1}-x\right) \tilde{u}^{\prime}+Q\left(2 x_{j+1}-x\right) \bar{u}=0 \text { on }\left[x_{j}{ }^{\prime}, x_{j+1}\right]
$$

with $\bar{u}\left(x_{j+1}\right)=0 ; \bar{u}^{\prime}\left(x_{j+1}\right)=u^{\prime}\left(x_{i+1}\right)$. Hence, by Theorem $5 /$ iii), we obtain $\mathrm{A}_{j+1} \subseteq A_{j}{ }^{\prime}$ if $-R\left(2 x_{j+1}-x\right) \geqq R(x)$ and $Q\left(2 x_{j+1}-x\right) \leqq Q(x), x \in\left[x_{j}{ }^{\prime}, x_{i+1}\right]$. By the assumptions on the coefficients in the present case these conditions are fulfilled. The remaining assertions of the theorem can analogously be proved

- By joining the Theorems 5 and 9 the following theorem is obtained.

Theorem 10: Let $u$ be an oscillatory solution to (26).

i) If $R$ (乡 0 and both functions $R$ and $Q$ are monotone decreasing (increasing), then

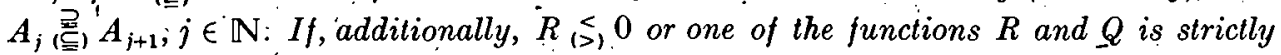

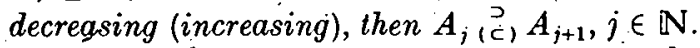

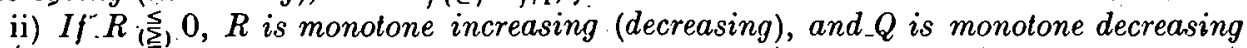

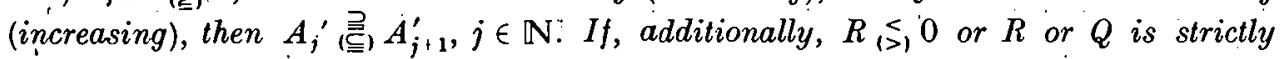
monotone increasing (decreasing), then $A_{j}^{\prime} \underset{(\subset)}{\vec{C}} A_{j+1}^{\prime}, j \in \mathbb{N}$.

Proof:- We handle the case that $\dot{R} \leqq 0$ and both functions $R$ and $Q$ are monotone decreasing. By Theorem $9 /$ ii), it follows that $A_{j}{ }^{\prime} A_{j+1}, j \in \mathbb{N}$. Hence, we have $\left|u\left(x_{j}{ }^{\prime}\right)\right| \geqq\left|u\left(x_{j+1}^{\prime}\right)\right|, j \in \mathbb{N}$. The function $v(x)=-u\left(x_{j+1}^{\prime}-x_{j}^{\prime}+x\right)$ is a solution to

$$
-\dot{v}^{\prime \prime}+R\left(x_{j+1}^{\prime}-x_{j}^{\prime}+x\right) v^{\prime}+Q\left(x_{j+1}^{\prime}-x_{j}^{\prime}+x\right) v=, 0 \text { on }\left[x_{j}, x_{j}^{\prime}\right]
$$

with $\left|v\left(x_{j}{ }^{\prime}\right)\right|=\left|u\left(x_{j+1}^{\prime}\right)\right| \leqq\left|u\left(x_{j}{ }^{\prime}\right)\right|$ and $v^{\prime}\left(x_{j}{ }^{\prime}\right)=-u^{\prime}\left(x_{j+1}^{\prime}\right)=0$. Since $R\left(x_{j+1}^{\prime}-x_{j}{ }^{\prime}+x\right)$ $\leqq \dot{R}(x)$ and $Q\left(x_{j+1}^{\prime}-x_{j}^{\prime}+x\right) \leqq Q(x), x \in\left[x_{i}, x_{j}^{\prime}\right]$, in view of Theorem 5/ii), it follows that $A_{j} \supseteqq A_{j+1}, j \in \mathbb{N}$. The remaining cases of the theorem can analogously be handled

Remark: Consider the differential equation $-u^{\prime \prime}+\varrho u^{\prime}+Q u=0 \quad(0>Q$ $\epsilon C[0, \infty), \varrho=$ const) and let $u$ be an oscillatory solution. Then by Theorems 9 and 10 the following holds:

i) If $\varrho \leqq 0$ and $Q$ is monotone decreasing, then $A_{j} \supseteqq A_{j+1}$ and $A_{j}^{\prime} \supseteqq A_{j+1}^{\prime}, j \in \mathbb{N}$. Hence, concerning the half-waves $A_{j} \cup A_{j}^{\prime}$ and $A_{j+1} \cup A_{j+1}^{\prime}$ ' we have $A_{j} \cup A_{j}^{\prime}$ $\supseteqq A_{j+1} \cup A_{j+1}^{\prime}$. If, additionally, $\varrho<0$ or $Q$ is strictly monotone decreasing, then $A_{j} \supset A_{j+1}$ and $A_{j}^{\prime} \supset A_{j+1}^{\prime}, j \in \mathbb{N}$.

ii) If $\varrho \geqq 0$ and $Q$ is monotone increasing, then $A_{j} \subseteq A_{j+1}$ and $A_{j}^{\prime} \subseteq A_{j+1}^{\prime}, j \in \mathbb{N}$. If, additionally, $\varrho>0$ or $Q$ is strictly monotone increasing, then $A_{j} \subset A_{j+1}$ and $A_{j}^{\prime}$, $\subset A_{j+1}^{\prime}, j \in \mathbb{N}$.

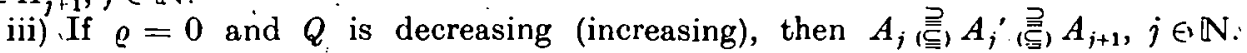
If $\varrho \doteq 0$ and $Q$ is strictly monotone decreasing (increasing), then $A_{j} \underset{(\subset)}{ } A_{j}^{\prime} \underset{(\subset)}{\supset} A_{j+1}$, $j \in \mathbb{N}$.

Concerning the selfadjoint equation

$$
-\left(P u_{-}^{\prime}\right)^{\prime}+Q u=0, \quad\left(0<P \in C^{1}[0, \infty), 0>Q \in C[0, \infty)\right)
$$

we have the following situation. 
Theorem 11: Let $u$ be an oscillatory solution to (27).

i) If $P$ is monotone increasing (decreasing) and $P^{-1} Q$ is monotone decreasing (increasing), then $A_{j}{ }^{\prime} \stackrel{\text { (롱) }}{A_{j+1}} \cdot j \in \mathbb{N}$. If, additionally, $P$ is strictly monotone increasing (decreasing) or $P^{-1} Q$ is strictly monotone decreasing (increasing), then $A_{j}^{\prime}\left(\vec{c}_{1} A_{j+1}\right.$, $\dddot{j \in \mathbb{N}}$.

ii) If $P, P^{-1} Q$ are monotone decreasing (increasing), then $A_{j}^{\prime}$ (ㅌ) $A_{j}, j \in \mathbb{N}$. If, additionally, $P$ or $P^{-1} Q$ is strictly monotone decreasing (increasing), then $A_{j}^{\prime}\left(\supset, A_{j}\right.$, $j \in \mathbb{N}$ :

iii) If $P, P^{-1} P^{\prime}$ are monotone increasing (decreásing), and $P^{-1} Q$ is monotone de-

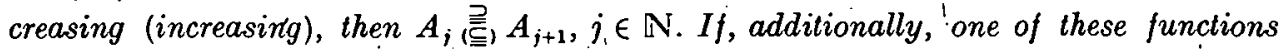
is strictly monotone, then $A_{j} \stackrel{\supset}{\text { (c) }} A_{j+1}, j \in \mathbb{N}$.

iv) If $P$ is monotone increasing (decreasing), and $P^{-1} P^{\prime}, P^{-1} Q$ are monotone decreasing, (increasing), then $A_{j}^{\prime} \cdot \stackrel{\text { ?륵 }}{A_{j+1}^{\prime}}, j \in \mathbb{N}$. If, additionally, one of these functions is strictly. monotone, then $A_{i}^{\prime}\left(\underset{\text { (C) }}{\supset} A_{j+1}^{\prime}, j \in \mathbb{N}\right.$ :

v) If $P(x)=\mathrm{e}^{c x}, c \underset{(\underline{\underline{z}})}{\gtrless}$, and $\mathrm{e}^{-c x} Q(x)$ is monotone decreasing (increasing), then

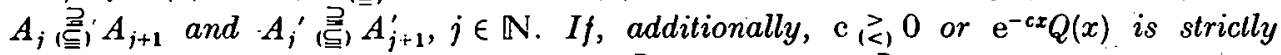

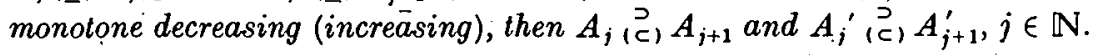

Proof: By considering that the equation (27) can be written as $-u^{\prime \prime}-P^{-1}$ $\times P^{\prime} u^{\prime}+P^{-1} Q=0$ Theorem 11 directly follows from the Theorems 9 and 10=

Finally, we apply the Theorems 9 and 10 to the Bessel differential equation

$$
-u^{\prime \prime}-x^{-1} u^{\prime}-\left(1-x^{-2} p^{2}\right) u=0, \quad x \in(0, \infty) \text {. }
$$

The Riccati differential equation (15) belonging to (28) calls

$$
y^{\prime}=y^{2}-x^{-1} y+\left(1-x^{-2} v^{2}\right), \quad x \in(0, \infty), \quad y=-u^{-1} u^{\prime}
$$

By means of (29) one can easily see that a non-trivial solution $u$ to (28) possesses at most one zero on $(0,|i|]$. Additionally, if the first zero $x_{1}$ of $u$ is placed in $(0,|v|)$, the first zero $x_{1}^{\prime}\left(>x_{1}\right)$ of $u^{\prime}$ is greater thán $|v|$. Hence, $Q(x)=-\left(1-x^{-2} v^{2}\right)<0, x$ $\in\left[x_{1}{ }^{\prime} ; \infty\right)$, and the Theorems $8-10$ can be applied to $(28)$ if $x$ is restricted by $x_{1}{ }^{\prime}$ $\leqq x<\infty$.

Theorem 12: Let $\mathscr{C}_{v}, v \neq 0$, be a non-trivial solution to (28) and denole its quarterwaves by $A_{j}$ and $A_{j}{ }^{\prime}, j \in \mathbb{N}$, respectively. Then, $A_{j}{ }^{\prime} \supset A_{j+1}$ and $A_{j}{ }^{\prime} \supset A_{j+1}^{\prime}, j \in \mathbb{N}$. Further we have $x_{j+1}-x_{j}^{\prime}>x_{j+1}^{\prime}-x_{j+1}, x_{j+1}-x_{j}^{\prime}>x_{j+2}-x_{j+1}^{\prime}$, and $\left|\mathscr{C}_{.}\left(x_{j}^{\prime}\right)\right|$ $>\left|\mathscr{C}_{v}\left(x_{j+1}^{\prime}\right)\right|, j \in \mathbb{N}$. In the special case $v=0$ the inclusions $A_{j}^{\prime} \supset A_{j+1}, A_{j}^{\prime} \supset A_{j+1}^{\prime}$, $A_{j+1}^{\prime} \supset A_{j+1}$ and the inequalities $x_{j+1}-x_{j}^{\prime}>x_{j+1}^{\prime}-x_{j+1}, x_{j+1}-x_{j}^{\prime}>x_{j+2}^{\prime}-x_{j+1}^{\prime}$, $x_{j+2}-x_{j+1}^{\prime}>x_{j+1}^{\prime}-x_{j+1},\left|\mathscr{C}_{0}\left(x_{j}^{\prime}\right)\right|>\left|\mathscr{C}_{0}\left(x_{j+1}^{\prime}\right)\right|, j \stackrel{j}{\epsilon} \mathbb{N}$, hold.

Proof: Apply the Theorems 9 and, 10

The inequalities $\left|\mathscr{C}_{v}\left(x_{j}{ }^{\prime}\right)\right|>\left|\mathscr{C}_{v}\left(x_{j+1}^{\prime}\right)\right|(\nu \in \mathbb{R}, j \in \mathbb{N})$ are due to L. Lorch, M. E. Muldoon, and P. Szegö. Additionally; they proved that the sequence $\left\{\mathscr{C}_{v}{ }^{2}\left(x_{j}{ }^{\prime}\right)\right\}_{j \in \mathbb{N}}$ is' completely monotonic (cf: [8])., Furthermore, they proved that the sequence $\left\{x_{j+1}^{\prime}-x_{j}{ }^{\prime}\right\}_{j \in \mathbb{N}}$ is also completely monotonic (cf. [8]).

Corollary: Assume $|\nu| \leqq 1 / 2$ and let $\mathscr{C}$, be a non-trivial solution to (28). Then, concerning, the zeros $x_{j}^{\prime}$ and $x_{j}^{\prime}$ of $\mathscr{C}_{v-}$ and $\mathscr{C}_{i}^{\prime}$, respectively, the inequalities $x_{j+1}-x_{j}{ }^{\prime}$ $>x_{j+1}^{\prime}-x_{j+1}, x_{j+1}-x_{j}^{\prime}>x_{j+2}-x_{j+1}^{\prime}$, and $x_{i+1}-x_{j}^{\prime}>x_{j}^{\prime}-x_{j}, j \in \mathbb{N}$, hold. 
Proof: In view of Theorem 12 we only have to show that $x_{j+1}-x_{j}^{\prime}>x_{j}^{\prime}-x_{j}$. It is well-known that $x_{j+1}-x_{j} \leqq x_{j+2}-x_{j+1}$ (cf. [7], for instance). Hence, together with the inequalities of Theorem 12, we obtain

$$
x_{j+1}-x_{j}^{\prime}+x_{j}^{\prime}-x_{j} \leqq x_{j+2}-x_{j+1}^{\prime}+x_{j+1}^{\prime}-x_{j+1}<2\left(x_{j+1}<x_{j}^{\prime}\right)
$$

and, consequently, the assertion $x_{j}^{\prime}-x_{j}<x_{j+1}-x_{j}^{\prime}, j \in \mathbb{N}$

\section{REFERENCES}

[1] Bifari, I.: Oscillation and monotonity theorems concerning non-linear differential equations of the second order. Acta Math. Acad. Sci. Hung. 9 (1958), 83-104.

[2] Hartman, P., and A. Wintner: On nonconservative linear oscillations of low frequency. Amer: J. Math. 70 (1948), 529-539.

[3] Kamke, E.: Differentialgleichungen reeller Funktionen. Leipzig: Geest \& Portig 1950.

[4] Laforgra, A.: Sturm theory for certain classes of Sturm-Liouville equations and Turanians and Wronskians for the zeros of derivative of Bessel functions. Nederl.'Akad. Wet. Indag. Math. 44 (1982), 295-301.

[5] Leighton, W.: Some elementary Sturm theory. J. Diff. Equ. 4 (1968), 187-193.

[6] Leichton, W.: More elementary Sturm theory. Appl.' Anal. 3 (1973), 187-203.

[7] Lorch, L., Muldoox, M., and P. Szeqö: Higher monotonicity propefties of certain Sturm. Liouville functions III. Can. J. Math. 22 (1970), 1238-1265.

[8] Lorch, L., Muldoon, M., and P. SzEGö: Higher monotonicity properties of certain SturmLiouville functions IV. Can. J. Math. 24 (1972), 349-368.

[9] MAKaI, E.: On a monotonic property of certain Sturm-Liouville functions. Acta Math. Akad. Sci. Hung. 3 (1952), 165-172.

[10] Müller-Pfeipfer, E.: Some remarks on the Sturmian theory for ordinary second order differential equations. Math. Nachr. (to appear):

Manuskripteingang: 28. 06. 1988; in revidierter Fassung 14.04. 1989

\section{VERFASSER:}

Prof. Dr. Erict MüLLER-Pfetffer

Sektion Mathematik/Ṕhysik der Pädag.' Hochschule „Dr. Th. Neubauer“

Nordhäuser Str. 63

DDR :5010 Erfurt 